\title{
The clinical benefit of molecular re-assessments in management of progressive lung cancer
}

\author{
Carina Wenzel $^{1,2}$, Sylvia Herold ${ }^{1,2}$, Felix C. Saalfeld ${ }^{2,3}$, Daniela E. Aust ${ }^{1,2}$, Martin Wermke ${ }^{2,3}$ \\ ${ }^{1}$ Institute for Pathology, University Hospital Carl Gustav Carus Dresden, TU Dresden, Germany; ${ }^{2}$ National Network Genomic Medicine Lung \\ Cancer (nNGM), Germany; ${ }^{3}$ Department for Internal Medicine I, University Hospital Carl Gustav Carus Dresden, TU Dresden, Germany \\ Correspondence to: PD Dr. med. Martin Wermke. Department for Internal Medicine I, University Hospital Carl Gustav Carus, Fetscherstraße 74, \\ 01307 Dresden, German. Email: martin.wermke@uniklinikum-dresden.de.
}

\begin{abstract}
Despite the enormous success of molecularly targeted therapy in advanced non-small cell lung cancer (NSCLC), long-term disease control remains challenging. Almost all patients on targeted therapy ultimately progress due to plethora of acquired resistance mechanisms. While acquired resistance mechanisms in BRAF-V600 mutant malignant melanomas treated with targeted therapy are well studied, little is known about resistance mechanisms in BRAF-V600 mutant lung cancer so far. Therefore, patients progressing on the standard BRAF and MEK inhibitor combination are uniformly switched to immune- and/ or chemotherapy. We describe the case of a metastatic BRAF-V600E mutant pulmonary adenocarcinoma of the left lung with presumed progression of a single lung lesion at the right side during targeted therapy. Due to oligo-progression, resection was performed. Molecular re-assessment for analysis of acquired resistance mechanisms surprisingly revealed a genetically distinct second primary malignancy. Following curative resection of the right sided second primary NSCLC, primary tyrosine kinase inhibitor therapy was continued and to date the patient is still responding with a cumulative treatment duration of now 34 months. This case report illustrates that a thorough molecular re-assessment upon progression on targeted therapies may have a decisive influence on subsequent treatment decisions and should therefore be considered on a routine basis.
\end{abstract}

Keywords: Case report; BRAF; non-small cell lung cancer (NSCLC); oligo-progression; molecular re-assessment

Submitted Aug 31, 2020. Accepted for publication Dec 23, 2020.

doi: $10.21037 /$ tlcr-20-996

View this article at: http://dx.doi.org/10.21037/tlcr-20-996

\section{Introduction}

Genomic analyses have resulted in a better understanding of the biology of non-small cell lung cancer (NSCLC) and in the successful development of molecularly targeted therapy $(1,2)$.

Somatic BRAF mutations occur in $2 \%$ to $4 \%$ of all NSCLC $(3,4)$, with approximately half of them showing a typical V600E mutation, associated with upregulation of MAPK signaling pathway (5). Even though monotherapy with BRAF-V600-specific inhibitors such as vemurafenib and dabrafenib shows clinical activity $(6,7)$, combination treatment with BRAF- and MEK-inhibitors results in even higher response and progression-free survival rates and has thus been licensed by FDA and EMA $(7,8)$.
In Melanoma, resistance to BRAF-inhibitor treatment is frequently caused by off-target mechanisms such as reactivation of MAPK signaling (9-11), PI3K-AKT pathway dysregulation and loss of CDKN2A $(12,13)$. On-target resistance mechanisms are less common in this setting with only a few publications describing BRAF amplifications (14) and alternative BRAF splicing variants (15) as potential mechanisms. Combinations of the described alterations may occur in melanoma patients treated with combined BRAF/ MEK inhibition (16).

In contrast, little is known about the mechanisms driving resistance to BRAF inhibitor or BRAF and MEK inhibitor treatment in NSCLC, with only a few case reports describing acquired KRAS, NRAS, MEK1 or PTEN mutations as potential off target mechanisms 

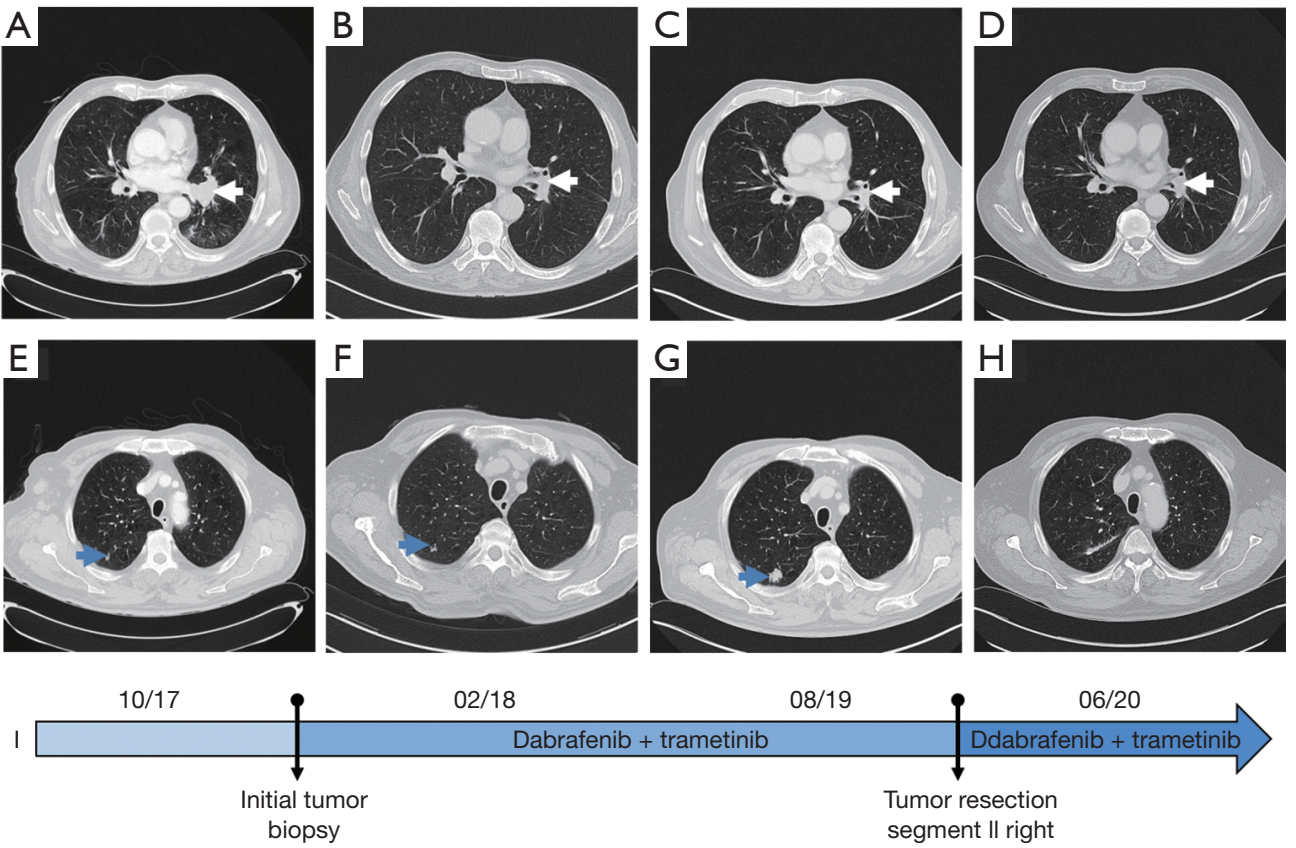

Figure 1 Clinical course of disease. Axial contrast-enhanced CT-scans of the primary tumor of the left lung (white arrow, top panel A,B,C,D) and the tumor mass in segment II of the right lung (blue arrow, bottom panel E,F,G,H) at different points in the follow-up and in correlation to patient's course of therapy (I).

(17-19). Therefore, current guidelines recommend to switch lung cancer patients progressing on first-line BRAF/ MEK inhibitor treatment to standard immune- and/or chemotherapy regimen (20).

In this case report, we describe the progressive disease of a patient with BRAF-V600E mutant metastatic adenocarcinoma of the left lung under combination therapy of dabrafenib and trametinib. We illustrate, that even in an apparently typical clinical course, a careful re-assessment of treatment options including renewed molecular diagnostic may be essential to optimize treatment outcome.

We present the following article in accordance with the CARE reporting checklist. Available at http://dx.doi. org/10.21037/tlcr-20-996.

\section{Case presentation}

In September 2017, a 75-year-old male patient with a 55 -year history of smoking presented with bone pain and increasing dyspnea. The initial computed tomography (CT) of thorax and abdomen showed a $33 \times 33 \mathrm{~mm}$ central mass in the left lower lobe of the lung (Figure 1) with multiple lymph node and bone metastases, which were histologically proven to represent a TTF1-positive pulmonary adenocarcinoma (Figure 2). Next-generation sequencing (NGS) revealed the presence of a BRAF-V600E mutation and no other targetable driver mutations.

Expression of PD-L1 was restricted to less than 1 percent of the tumor cells.

Thus, a combination of dabrafenib (150 mg twice daily orally) and trametinib ( $2 \mathrm{mg}$ /day orally) was started and resulted in partial response (PR) in subsequent CT examinations (decrease of the index lesion to 24 $\times 18 \mathrm{~mm}$ in Figure $1 B$ ). However, a single lesion in segment II of the contralateral lung, which was hardly visible in the initial CT-scan, progressed in size (to $20 \times 15 \mathrm{~mm}$ in Figure $1 G$ ) after 18 months of treatment. At that point, we considered switching to immunochemotherapy. However, as the lesion in segment II (right) was the only progressive tumor manifestation and the patient was reluctant to start a chemotherapy containing regimen resection of this single lesion was offered. Histology demonstrated TTF1positive adenocarcinoma (Figure $2 F, G, H, I$ ) compatible with the primary tumor. In an attempt to elucidate potential resistance mechanisms, we performed NGS-based panel sequencing. Much to our surprise, we were unable to find the initial BRAF-V600E mutation. Therefore, we performed additional sequencing on both tumor samples 

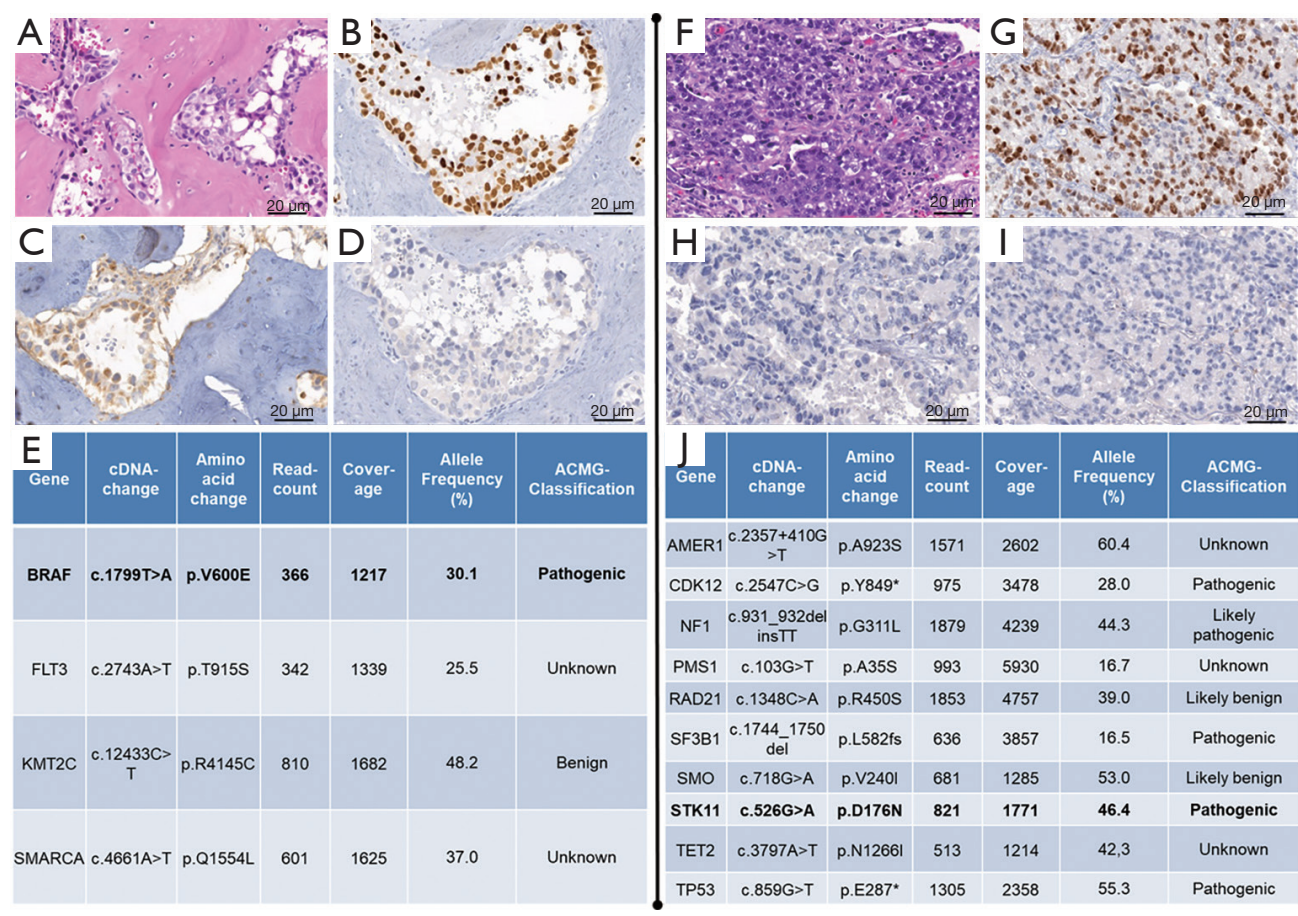

Figure 2 Histological and molecular findings. Initial diagnosis of a solid pulmonary adenocarcinoma (A) with immunohistochemical expression of TTF1 (B) and without expression of p40 (C) and synaptophysin (D). Results of NGS analyses of the initial tumor with detection of an activating BRAF V600E mutation and further single nucleotide variants in other genes (E). Histology of the tumor in segment II of the right lung with detection of a solid adenocarcinoma (F) with immunohistochemical expression of TTF1 (G) and without expression of p40 (H) and synaptophysin (I). NGS analyses of the tumor in segment II of the right lung with detection of several divergent genetic variants and without detection of a BRAF V600E mutation (J). The annotation of the detected variants was done in accordance to ACMG classification (21); (I) benign, (II) likely benign, (III) uncertain significance, (IV) likely pathogenic, (V) pathogenic. The following reference transcripts were used: AMER1 (NM_152424.3), BRAF (NM_004333.4), CDK12 (NM_016507.3), FLT3 (NM_004119.2), KMT2C (NM_170606.2), NF1 (NM_001042492.2), PMS1 (NM_000534.4), RAD21 (NM_006265.2), SF3B1 (NM_012433.3), SMARCA (NM_001128849.2), SMO (NM_005631.4), STK11 (NM_000455.4), TET2 (NM_001127208.2), TP53 (NM_001276760.1).

with identical commercially available, larger amplicon based NGS panels (Human Comprehensive Cancer Panel and Human Oncology Panel, Qiagen) for comprehensive molecular characterization and comparison of both specimen.

The regions of interest were amplified using the above mentioned amplicon panels according to the protocol "QIAseq Targeted DNA V3 Panel, May 2017" (Qiagen). Tumor cell content of both samples was at least $70 \%$. The bioinformatics evaluation was performed with Biomedical Workbench from CLC (12.0.3) using a customized analysis algorithm with the following filters: coverage $>/=200$, allele frequency $>/=10 \%$. HG19 was used as reference genome.

The initial left-sided tumor (Figure 2E) and the resected right-sided lesion (Figure 2f) showed completely distinct molecular profiles with not a single shared variant, arguing for the latter being a second independent BRAF-wildtype NSCLC rather than a metastasis of the initial BRAFmutated cancer.

Given the complete resection of the second primary BRAF-wildtype NSCLC, the well-tolerated and effective TKI combination therapy was continued. The patient remained in stable PR at last follow-up, 34 months after treatment initiation and 12 months after resection of the single progressing lesion in segment II right (index lesion $26 \times 16 \mathrm{~mm}$; Figure 1D).

\section{Ethical statement}

All procedures performed in this study were in accordance 
with the ethical standards of the institutional and national research committee and with the Helsinki Declaration (as revised in 2013). Written informed consent was obtained from the patient.

\section{Discussion}

Almost $60 \%$ of all patients with NSCLC harbor an oncogenic driver mutation with nearly half of them being therapeutically targetable (1). Targeted therapies have, in comparison to conventional chemotherapy, resulted in impressive improvements in response rates, survival and patient-reported quality of life (22). However, the treatment of advanced NSCLC with typical driver mutations remains a challenge due to the invariable development of on- and off-target resistance (23).

While molecular re-assessment guided selection of targeted second-line treatment has been established for more frequent molecular subtypes of NSCLC $(24,25)$, BRAF-mutated patients progressing on BRAF/MEKinhibition are usually switched to immuno(chemo) therapy (20). This seems reasonable as response rates to immuno(chemo)therapy in BRAF-mutated patients are comparable to the non-mutated situation (26-28). However, an increase in the frequency of re-biopsies with renewed molecular diagnostic will be necessary to elucidate and potentially target resistance mechanism in this rare mutation subtype. Our case illustrates that this is of special importance in patients with "oligo-progression", which may represent local clonal evolution $(29,30)$ but also the emergence of a second primary malignancy. Even though the genetic information obtained for both tumors is limited by the size of the applied NGS-panels, we believe that the grade of molecular disparity clearly argues against clonal evolution in our case. The STK11 mutation identified in the second BRAF-wildtype NSCLC of our patient has been associated with decreased activity of immune checkpoint-inhibitor based treatment. Therefore following the current clinical standard by switching to this treatment modality might not have had the desired effect in this individual (31).

Keeping in mind the limitations of a single case report, we think that the course of our patient illustrates, that molecular re-assessment of tumor material obtained at time of progression might have a tremendous impact on an individual patient's outcome and should therefore be considered on a routine basis.

\section{Acknowledgments}

Funding: This work was supported by the Stiftung Deutsche Krebshilfe within the National Network Genomic Medicine Lung Cancer, nNGM.

\section{Footnote}

Reporting Checklist: The authors have completed the CARE reporting checklist. Available at http://dx.doi.org/10.21037/ tlcr-20-996

Conflicts of Interest: All authors have completed the ICMJE uniform disclosure form (Available at http://dx.doi. org/10.21037/tlcr-20-996). FCS reports personal fees from Takeda Pharma Vertrieb GmbH und Co. KG, personal fees from Pfizer Pharma GmbH, non-financial support from Eli Lilly and Company, outside the submitted work. DEA reports personal fees from Astra Zeneca, personal fees from Roche, personal fees from MSD, outside the submitted work. MW reports personal fees and non-financial support from Novartis, outside the submitted work. The other authors have no conflicts of interest to declare.

Ethical Statement: The authors are accountable for all aspects of the work. The authors ensure that questions related to the accuracy or integrity of any part of the work were appropriately investigated and resolved. All procedures performed in this study involving human participants were in accordance with the ethical standards of the institutional and national research committee and with the Helsinki Declaration (as revised in 2013). Written informed consent was obtained from the patient.

Open Access Statement: This is an Open Access article distributed in accordance with the Creative Commons Attribution-NonCommercial-NoDerivs 4.0 International License (CC BY-NC-ND 4.0), which permits the noncommercial replication and distribution of the article with the strict proviso that no changes or edits are made and the original work is properly cited (including links to both the formal publication through the relevant DOI and the license). See: https://creativecommons.org/licenses/by-nc-nd/4.0/.

\section{References}

1. Herbst RS, Morgensztern D, Boshoff C. The biology and management of non-small cell lung cancer. Nature 
2018;553:446-54.

2. Clinical Lung Cancer Genome Project (CLCGP); Network Genomic Medicine (NGM). A Genomics-Based Classification of Human Lung Tumors. Sci Transl Med 2013;5:209ra153.

3. Cardarella S, Ogino A, Nishino M, et al. Clinical, Pathologic, and Biologic Features Associated with BRAF Mutations in Non-Small Cell Lung Cancer. Clin Cancer Res 2013;19:4532-40.

4. Paik PK, Arcila ME, Fara M, et al. Clinical Characteristics of Patients With Lung Adenocarcinomas Harboring BRAF Mutations. J Clin Oncol 2011;29:2046-51.

5. Yao Z, Yaeger R, Rodrik-Outmezguine VS, et al. Tumours with class 3 BRAF mutants are sensitive to the inhibition of activated RAS. Nature 2017;548:234-8.

6. Hyman DM, Puzanov I, Subbiah V, et al. Vemurafenib in multiple nonmelanoma cancers with BRAF V600 mutations. N Engl J Med 2015;373:726-36.

7. Planchard D, Kim TM, Mazieres J, et al. Dabrafenib in patients with BRAFV600E-positive advanced non-smallcell lung cancer: A single-arm, multicentre, open-label, phase 2 trial. Lancet Oncol 2016;17:642-50.

8. Planchard D, Smit EF, Groen HJM, et al. Dabrafenib plus trametinib in patients with previously untreated BRAFV600E-mutant metastatic non-small-cell lung cancer: an open-label, phase 2 trial. Lancet Oncol 2017;18:1307-16.

9. Wagle N, Emery C, Berger MF, et al. Dissecting Therapeutic Resistance to RAF Inhibition in Melanoma by Tumor Genomic Profiling. J Clin Oncol 2011;29:3085-96.

10. Nazarian R, Shi H, Wang Q, et al. Melanomas acquire resistance to B-RAF(V600E) inhibition by RTK or N-RAS upregulation. Nature 2010;468:973-7.

11. Nissan MH, Pratilas CA, Jones AM, et al. Loss of NF1 in cutaneous melanoma is associated with RAS activation and MEK dependence. Cancer Res 2014;74:2340-50.

12. Paraiso KHT, Xiang Y, Rebecca VW, et al. PTEN Loss Confers BRAF Inhibitor Resistance to Melanoma Cells through the Suppression of BIM Expression. Cancer Res 2011;71:2750-60.

13. Shi H, Hugo W, Kong X, et al. Acquired Resistance and Clonal Evolution in Melanoma during BRAF Inhibitor Therapy. Cancer Discov 2014;4:80-93.

14. Shi H, Moriceau G, Kong X, et al. Melanoma wholeexome sequencing identifies V600E B-RAF amplificationmediated acquired B-RAF inhibitor resistance. Nat Commun 2012;3:724.
15. Poulikakos PI, Persaud Y, Janakiraman M, et al. RAF inhibitor resistance is mediated by dimerization of aberrantly spliced BRAF(V600E). Nature 2011;480:387-90.

16. Moriceau G, Hugo W, Hong A, et al. TunableCombinatorial Mechanisms of Acquired Resistance Limit the Efficacy of BRAF/MEK Cotargeting but Result in Melanoma Drug Addiction. Cancer Cell 2015;27:240-56.

17. Rudin CM, Hong K, Streit M. Molecular Characterization of Acquired Resistance to the BRAF Inhibitor Dabrafenib in a Patient with BRAF-Mutant Non-Small-Cell Lung Cancer. J Thorac Oncol 2013;8:e41-2.

18. Niemantsverdriet M, Schuuring E, Elst AT, et al. KRAS Mutation as a Resistance Mechanism to BRAF/MEK Inhibition in NSCLC. J Thorac Oncol 2018;13:e249-51.

19. Facchinetti F, Lacroix L, Mezquita L, et al. Molecular mechanisms of resistance to BRAF and MEK inhibitors in BRAFV600E non-small cell lung cancer. Eur J Cancer 2020;132:211-23.

20. NCCN. NCCN Clinical Practice Guidelines in Oncology. 2020. Available online: https://www.nccn.org/ professionals/physician_gls/pdf/nscl_blocks.pdf

21. Richards S, Aziz N, Bale S, et al. Standards and guidelines for the interpretation of sequence variants: A joint consensus recommendation of the American College of Medical Genetics and Genomics and the Association for Molecular Pathology. Genet Med 2015;17:405-24.

22. von Verschuer U, Schnell R, Tessen HW, et al. Treatment, outcome and quality of life of 1239 patients with advanced non-small cell lung cancer - final results from the prospective German TLK cohort study. Lung Cancer 2017;112:216-24.

23. Rotow J, Bivona TG. Understanding and targeting resistance mechanisms in NSCLC. Nat Rev Cancer 2017;17:637-58.

24. Jänne PA, Chih-Hsin Yang J, Kim DW, et al. AZD9291 in EGFR inhibitor-resistant non-small-cell lung cancer. N Engl J Med 2015;372:1689-99.

25. Gainor JF, Dardaei L, Yoda S, et al. Molecular mechanisms of resistance to first- and second-generation ALK inhibitors in ALK -rearranged lung cancer. Cancer Discov 2016;6:1118-33.

26. Borghaei H, Paz-Ares L, Horn L, et al. Nivolumab versus Docetaxel in Advanced Nonsquamous Non-Small-Cell Lung Cancer. N Engl J Med 2015;373:1627-39.

27. Mazieres J, Drilon A, Lusque A, et al. Immune checkpoint inhibitors for patients with advanced lung cancer and oncogenic driver alterations: Results from the IMMUNOTARGET registry. Ann Oncol 2019;30:1321-8. 
28. Guisier F, Dubos-Arvis C, Viñas F, et al. Efficacy and Safety of Anti-PD-1 Immunotherapy in Patients With Advanced NSCLC With BRAF, HER2, or MET Mutations or RET Translocation: GFPC 01-2018. J Thorac Oncol 2020;15:628-36.

29. Jamal-Hanjani M, Wilson GA, McGranahan N, et al. Tracking the Evolution of Non-Small-Cell Lung Cancer. N Engl J Med 2017;376:2109-21.

30. Califano R, Romanidou O, Mountzios G, et al.

Cite this article as: Wenzel C, Herold S, Saalfeld FC, Aust DE, Wermke $M$. The clinical benefit of molecular re-assessments in management of progressive lung cancer. Transl Lung Cancer Res 2021;10(3):1582-1587. doi: 10.21037/tlcr-20-996
Management of NSCLC Disease Progression after FirstLine EGFR Tyrosine Kinase Inhibitors: What Are the Issues and Potential Therapies? Drugs 2016;76:831-40.

31. Koyama S, Akbay EA, Li YY, et al. STK11/LKB1 Deficiency Promotes Neutrophil Recruitment and Proinflammatory Cytokine Production to Suppress T-cell Activity in the Lung Tumor Microenvironment. Cancer Res 2016;76:999-1008. 